\title{
Obesity affects skeletal muscle ketone oxidation
}

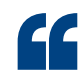

\section{mice treated with pimozide had reduced circulating levels of glucose compared with control mice} g metabolism, in particular, ketone oxidation," explains corresponding author John Ussher. "With more of the world's population willing to consider ketogenic dieting as a nutritional approach for weight loss in obesity, we thought it would be prudent to further understand how obesity itself might influence ketone oxidation."

During periods of fasting, the liver produces ketone bodies (for example, $\beta$-hydroxybutyrate $(\beta \mathrm{OHB})$ and acetoacetate), which are oxidized by the brain as an alternative fuel source to glucose. Other tissues, such as skeletal muscle and cardiac tissue, can also

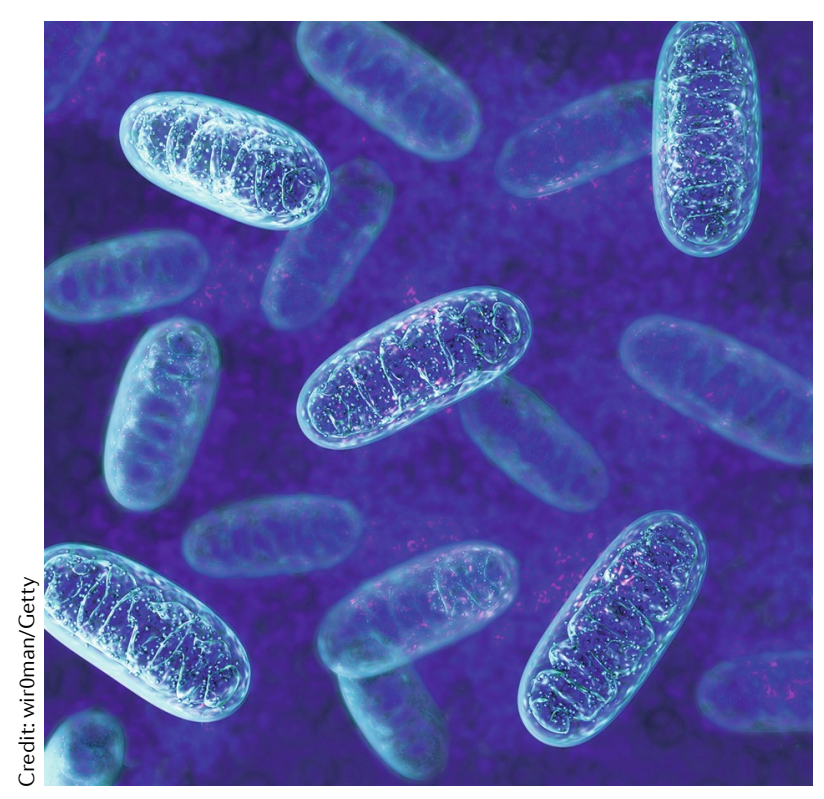

oxidize ketone bodies. Of note, the rate-limiting enzyme for ketone body oxidation is succinyl-CoA:3 ketoacid coenzyme A transferase 1 (SCOT, encoded by OXCT1).

Ussher and colleagues investigated metabolism in a mouse model of diet-induced obesity. Notably, compared with lean mice, obese mice had substantially lower circulating levels of $\beta \mathrm{OHB}$, with corresponding increases in SCOT expression and activity in skeletal muscle. To investigate whether these obesity-associated changes were harmful or beneficial, the researchers undertook a virtual high-throughput screening programme to find potential antagonists of SCOT. The screen identified pimozide, an agent that was originally developed as an antipsychotic and is FDAapproved for controlling tics in individuals with Tourette syndrome, as a potential drug of interest on the basis of binding energy and binding interaction types. In vitro assays using myotubes and ex vivo assays with isolated working mouse hearts confirmed the activity of pimozide as a SCOT antagonist.

Next, obese male mice were treated with pimozide or a vehicle control once every 2.5 days for 2 weeks. Notably, mice treated with pimozide had reduced circulating levels of glucose compared with control mice, suggesting that the increase in SCOT activity in obesity could be harmful. Further studies confirmed that the reduction in hyperglycaemia observed in pimozide-treated animals was independent of oxidative stress, lipid accumulation and the activity of SCOT in the brain.

To confirm the harmful effects of increased SCOT activity and skeletal muscle ketone oxidation in obesity, Ussher and colleagues generated a skeletal muscle-specific
OXCT1-knockout mouse (SCOT $^{\text {Muscle-/-) }}$. Control mice and SCOT ${ }^{\text {Muscle-/- }}$ mice were fed a high-fat diet to induce obesity. Importantly, obese SCOT ${ }^{\mathrm{Muscle}-/-}$ mice had reduced hyperglycaemia compared with obese control mice, thereby confirming the harmful effects of increased skeletal muscle ketone body oxidation in obesity. Obese SCOT $^{\text {Muscle-l- }}$ mice also had improved glucose tolerance following an overnight fast. Of note, the improvements in glucose metabolism seen in pimozide-treated obese control mice were not seen in pimozide-treated obese SCOT ${ }^{\text {Muscle-l- }}$ mice, confirming the requirement for skeletal muscle SCOT for the activity of pimozide.

Finally experiments in both untreated obese SCOT ${ }^{\text {Muscle-/- }}$ mice and pimozide-treated obese control mice showed that the mechanism for glycaemic improvement following SCOT antagonism involved increased activity of pyruvate dehydrogenase, the rate-limiting enzyme of glucose oxidation.

"Because pimozide is already approved for use in humans, we hope to work together with clinical researchers to determine whether our findings translate to humans with obesity and/or T2DM, to confirm whether pimozide can be repurposed for these individuals," concludes Ussher. "Ideally we would design pimozide-like compounds that do not cross the blood-brain barrier, in order to preserve ketones as a fuel for the brain, while inhibiting it peripherally in the skeletal muscle to control high blood sugar levels in people with T2DM."

Shimona Starling

ORIGINAL ARTICLE Al Batran, R. et al. Pimozide alleviates hyperglycemia in diet-induced obesity by inhibiting skeletal muscle ketone oxidation. Cell Metab. https://doi.org/10.1016/j.cmet.2020. 03.017 (2020) 